\title{
Nonlocal Thermodynamics: Mathematical Model of Two-Dimensional Thermal Conductivity
}

\author{
George Kuvyrkin ${ }^{1}$, Inga Savelyeva ${ }^{1}$, and Daria Kuvshinnikova ${ }^{1}$ \\ ${ }^{1}$ Department of Applied Mathematics, Bauman Moscow State Technical University, 105005, 2nd Baumanskaya st 5, Moscow, \\ Russian Federation
}

\begin{abstract}
Nonlocal models of thermodynamics are becoming more and more popular in the modern world. Such models make it possible to describe materials with a complex structure and unique strength and temperature properties. Models of nonlocal thermodynamics of a continuous medium establish a relationship between micro and macro characteristics of materials. A mathematical model of thermal conductivity in nonlocal media is considered. The model is based on the theory of nonlocal continuum by A.K. Eringen. The interaction of material particles is described using local and nonlocal terms in the law of heat conduction. The nonlocal term describes the effect of decreasing the influence of the surrounding elements of the material structure with increasing distance. The effect of nonlocal influence is described using the standard non-locality function based on the Gaussian distribution. The nonlocality function depends on the distance between the elements of the material structure. The mathematical model of heat conduction in a nonlocal medium consists of an integro-differential heat conduction equation with initial and boundary conditions. A numerical solution to the problem of heat conduction in a nonlocal plate is obtained. The numerical solution of a two-dimensional problem based on the finite element method is described. The influence of nonlocal effects and material parameters on the thermal conductivity in a plate under highintensity surface heating is analyzed. The importance of nonlocal characteristics in modelling the thermodynamic behaviour of materials with a complex structure is demonstrated.
\end{abstract}

\section{Nomenclature}

$\begin{array}{ll}c & - \text { volumetric heat generation density, } \\ & {[\mathrm{J} / \mathrm{kg} * \mathrm{~K}]} \\ p_{1}, p_{2} & - \text { local and nonlocal component weights, [-] } \\ & p_{1}+p_{2}=1 . \\ q & - \text { one dimensional heat flow, }\left[\mathrm{W} / \mathrm{m}^{2}\right] \\ T & - \text { temperature, }[\mathrm{K}] \\ t & - \text { time, }[\mathrm{s}] \\ V & - \text { scope of influence, }\left[\mathrm{m}^{3}\right] \\ x & - \text { space coordinate, }[\mathrm{m}] \\ \rho & - \text { material density, }\left[\mathrm{kg} / \mathrm{m}^{3}\right] \\ a & - \text { parameter of the non-local material, }[-] \\ \kappa^{(T)} & - \text { dimensionless coefficients of thermal } \\ \theta & \text { diffusivity, }[-] \\ z & - \text { dimensionless temperature, }[-] \\ \text { Superscripts } & - \text { dimensionless space coordinate, }[-] ; \\ (n l) & - \text { nonlocal vector }\end{array}$

\section{Introduction}

The classical theory of continuum is not suitable for the mechanical analysis of micro- and nanostructures, since it is scaleless and does not have parameters for the scale of structural elements. On the other hand, sizedependent theories of thermomechanics such as surface stress theory [1-2], gradient theory [3] and nonlocal theory [4-7] are widely used to study the mechanical behavior of materials at the micro and nanoscale due to their ability to take into account the effect of size. Among them, the nonlocal theory developed by Ehringen and his collaborators [7] is the well-known nonclassical theory of thermoelasticity, in which the behavior at a material point depends on the state of all points of the body. Ehrengen's theory uses a nonlocality influence function (decaying with distance), which includes a length scale parameter. This approach allows one to obtain intego-differential nonlocal constitutive equations that take into account interatomic interactions.

Eringen's theory of nonlocality has been widely developed among scientists around the world. The authors of this article considered one-dimensional thermomechanical problems, devoted to the issues of thermomechanical behavior of structures [8-13]. The next stage of research is the solution of two-dimensional problems describing the distribution of stresses and temperature fields in two-dimensional regions. In this article, the emphasis will be made on the analysis of temperature fields in two-dimensional plates under various conditions of external loading. The non-local approach is based on the idea that the heat flux at a control point is a function of the macroscopic field of the temperature gradient at all points of the body. The general governing equations of nonlocal heat conduction are expressed through the nonlocal heat flux [6] 


$$
\begin{aligned}
q_{i}^{(n l)}(\mathbf{x}, t)=-p_{1} \lambda_{i j}^{(T)} & \frac{\partial T(\mathbf{x}, t)}{\partial x_{j}}- \\
& -p_{2} \lambda_{i j}^{(T)} \int_{V} \varphi\left(\left|\mathbf{x}^{\prime}-\mathbf{x}\right|\right) \frac{\partial T\left(\mathbf{x}^{\prime}, t\right)}{\partial x_{j}^{\prime}} d x_{j}{ }^{\prime} .
\end{aligned}
$$

Substituting (1) into the heat conduction equation in vector form [8], we obtain:

$$
\rho c \frac{\partial T}{\partial t}=-\frac{\partial q_{i}}{\partial x_{i}}+q_{V}, \quad i=\overline{1,3}
$$

The law of heat propagation in a medium, taking into account the effect of spatial nonlocality:

$$
\begin{aligned}
\rho c \frac{\partial T}{\partial t} & =p_{1} \frac{\partial}{\partial x_{i}} \lambda_{i j}^{(T)} \frac{\partial T(\mathbf{x}, t)}{\partial x_{j}}+ \\
& +p_{2} \lambda_{i j}^{(T)} \frac{\partial}{\partial x_{i}} \int_{V} \varphi\left(\left|\mathbf{x}^{\prime}-\mathbf{x}\right|\right) \frac{\partial T\left(\mathbf{x}^{\prime}, t\right)}{\partial x_{j}^{\prime}} d x_{j}^{\prime} .
\end{aligned}
$$

This mathematical model allows one to take into account two existing opposing concepts of describing the structure of any rigid body - the concept of continuity and discreteness. The discreteness is taken into account by introducing the nonlocality influence function $\varphi\left(\left|\mathbf{x}^{\prime}-\mathbf{x}\right|, a\right)$, which depends on the distance between the current point and the nearest neighbors, as well as on the nonlocal parameter $a$. The parameter of a nonlocal material should be determined experimentally by matching the acoustic dispersion curves of the material. The nonlocality function of the form [7] is used as standard:

$$
\varphi\left(\left|\mathbf{x}^{\prime}-\mathbf{x}\right|\right)=\frac{1}{2 a} \exp \left(-\frac{\left|\mathbf{x}^{\prime}-\mathbf{x}\right|}{a}\right), \quad\left|\mathbf{x}^{\prime}-\mathbf{x}\right|<a .
$$

Equations (3) - (4) with boundary conditions define an integro-differential formulation of nonlocal heat conduction in a solid.

\section{Analysis and modelling}

Consider a two-dimensional domain $S$ with a piecewise smooth boundary $\partial S$. The equation of thermal conductivity (3) in dimensionless form will take the form:

$$
\begin{aligned}
& \frac{\partial \theta}{\partial t}=p_{1} \frac{\partial}{\partial z_{i}} \kappa_{i j}^{(T)} \frac{\partial \theta(\mathbf{z}, t)}{\partial z_{j}}+ \\
& \quad+p_{2} \kappa_{i j}^{(T)} \frac{\partial}{\partial z_{i}} \int_{S^{\prime}\left(z^{\prime}\right)} \varphi\left(\left|\mathbf{z}^{\prime}-\mathbf{z}\right|\right) \frac{\partial \theta\left(\mathbf{z}^{\prime}, t\right)}{\partial z_{j}^{\prime}} d S^{\prime}\left(\mathbf{z}^{\prime}\right) .
\end{aligned}
$$

where $i=1,2, S^{\prime}(\mathbf{z})$ is the area of non-local influence, $\mathbf{z}^{\prime} \in S^{\prime}(\mathbf{z})$.

The first term in equation (5), with a weighting factor $p_{1}$, is responsible for the contribution of the local (classical) component of the heat flux function described by the Bio-Fourier law [8]. The second term, integral with weight $p_{2}$, describes the effect of the nonlocal action of points in the area $S^{\prime}(\mathbf{z})$ on the point $\mathbf{z}$ considered at a given moment.

Consider a plate $S$. Boundary conditions of the second kind are specified on the lateral faces of the plate:

$$
\left.q^{(n l)}(\mathbf{z}, t) \cdot \mathbf{n}\right|_{z_{2}=0}=\left.q^{(n l)}(\mathbf{z}, t) \cdot \mathbf{n}\right|_{z_{2}=1}=0 .
$$

A constant temperature $\left.\theta\right|_{z_{1}=1}=\theta_{0}$. is set at the upper limit.

At the lower limit, a heat flux is set:

$$
\left.q^{(n l)}(\mathbf{z}, t) \cdot \mathbf{n}\right|_{z_{1}=0}=q_{0}\left(z_{1}\right) .
$$

Equation (5) with boundary conditions (6) - (7) describes the process of heat propagation taking into account nonlocal effects in a two-dimensional plate.

To solve equation (5) with boundary conditions (6) - (7), we use the finite element method. Let us introduce a finite element model grid $S_{h}$ in the domain $S(\mathbf{z})$, then equation (5) can be represented as a system of linear algebraic equations [14], the solution of which is possible using the Seidel method [15].

\section{Results and discussion}

Consider a plate $S=[0 ; 1] \times[0 ; 1]$. Thermal insulation conditions are specified on the side faces (6). At the upper boundary, a constant temperature $\theta_{0}=1$ is set, at the lower boundary, a flow with an intensity $I=50000$ and an impact area $h=0.02$ is specified, which corresponds to a heat flow $q_{0}=1000$. Heating a small area on the edge of the plate simulates the high-intensity heating of a structural element by a laser beam. Figure 1 shows a comparison of the contour plots of the temperature field distribution in the plate for different weights of the local component $p$ (parameter $p_{1}$ from equation (5)). The red line denotes the contour plot of the temperature field when solving the problem of classical parabolic heat conduction, without taking into account the contribution of nonlocality. We see that with a significant contribution of nonlocality, $p=0.2$ the yellow line in Fig. 1, the propagation of heat and the leveling of the solution profile occurs much faster than in the classical case.

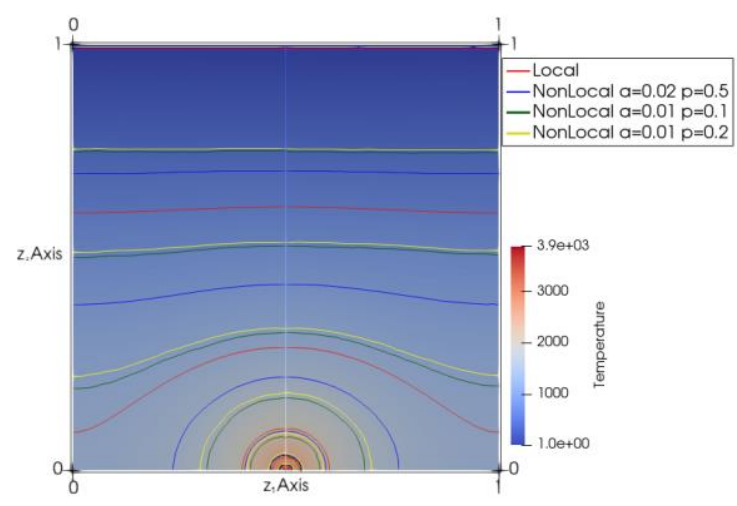

Fig. 1. Influence of the parameter of the contribution of the local component on the temperature distribution in the plate

Figure 2 shows the temperature distribution in the central section of the plate (Fig. 1), perpendicular to the bottom face. The graph of the temperature distribution in the central section shows how the distribution changes depending on the weight of the local component $p$ and the parameter of the non-local material $a$. The 
classical solution of parabolic thermal conductivity without taking into account the effect of nonlocality is shown in blue. We see that with an increase in the parameter of the non-local material $a$, the solution to the non-local problem becomes similar to the classical one (comparison of the red, blue and pink curves in Figure 2). This is due to the fact that a larger number of material structures fall into the zone of influence of nonlocality. At the same time, with a larger parameter $a$ and equal weight $p$ (red and pink curves in Fig. 2), it can be seen that the temperature value at the upper boundary of the plate is higher than with a smaller parameter of the nonlocal material (pink curve in Fig. 2).

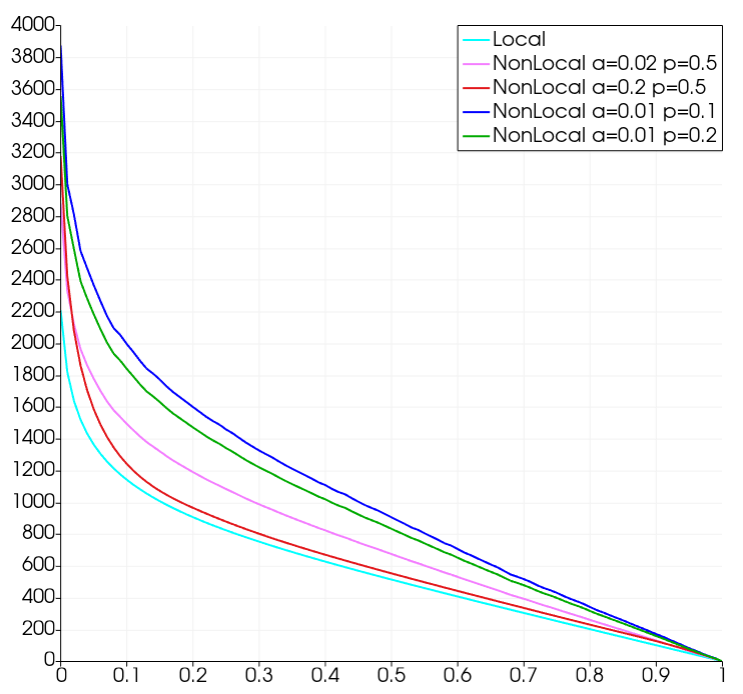

Fig. 2. Temperature distribution in the section $z_{1}=0.5$

Figure 3 shows the temperature distribution in a section parallel to the lower boundary of the plate (at a distance $z_{2}=0.01$ from the lower boundary of the plate). Figure 3 shows the temperature distribution in a section close to the lower edge of the plate, which is subject to high-intensity impact. We see that the greatest absorption of the impact occurs by the material with the nonlocality parameters $a=0.001$ and $p=0.2$ (green curve in Fig. 3). This case corresponds to a material with the most pronounced nonlocal properties and indicates the importance of taking into account the structure when studying the propagation of heat in structure-sensitive materials.

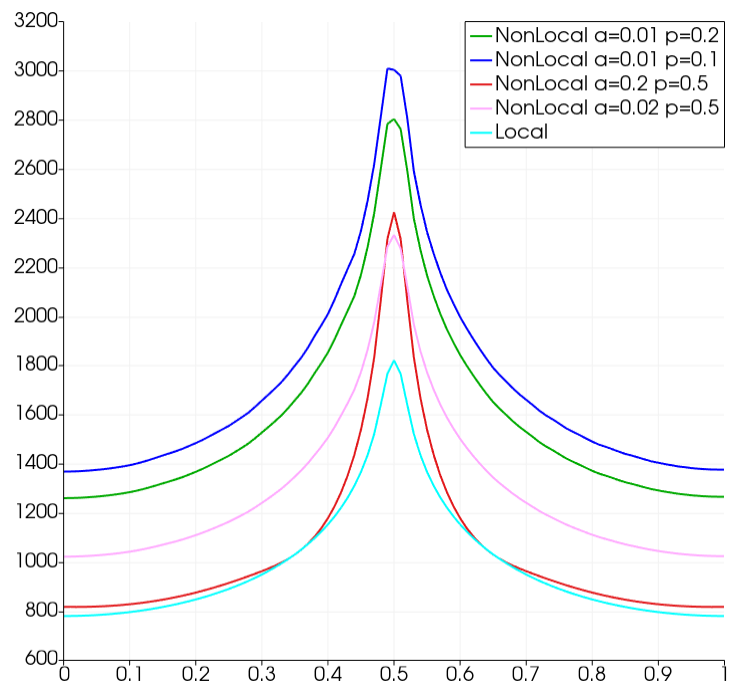

Fig. 3. Temperature distribution in the section $z_{2}=0.01$

\section{Conclusions}

A mathematical model of heat propagation in non-local media is considered. Numerical solutions are obtained for the problem of heat propagation in a twodimensional plate heated by a high-intensity point heat flow. The influence of the nonlocal parameters of the material on the heat propagation in the plate under highintensity surface heating is analyzed. It is shown that taking into account size effects and long-range forces is important when modeling materials with a complex structure.

Work of I.Yu. Savelyeva is supported by Russian Science Foundation (proj. 17-79-20445).

\section{References}

1. R. A. Andrievskiy, G. Ros. chem. J. Society for them. D.I. Mendeleyev, 46(5), pp.50-56 (2002).

2. R. A. Andrievskiy, A. M. Glezer, Phys.Usp., 52(4), pp. 315-334 (2009).

3. E. Roduner, J. Chem Soc Rev, 35(5), pp. 83-92 (2006).

4. A. A. Pisano, P. Fuschi, International journal of Solids and Structures, 40(1), pp. 13-23 (2003).

5. C. Polizzotto, Int. J. Solids Struct, 38, pp. 73597380 (2001).

6. G. N. Kuvyrkin, I. Y. Savelyeva, D. A. Kuvshynnikova, Journal of Physics: Conference Series, 991(1), 012050 (2018).

7. A. C. Eringen, Nonlocal Continuum Field Theories (2002).

8. V. S. Zarubin, G. N. Kuvyrkin, Mathematical Models of Continuum Mechanics and Electrodynamics (2008).

9. G. N. Kuvyrkin, I. Yu. Svelyeva, A. V. Zhuravskii, Heat and Mass Transfer, 56(2), pp. 423-427 (2020). 
10. A. V. Cherednichenko, I. Yu. Savelyeva and A. P. Shukhtin, AIP Conference Proceedings, 2293, Art.no 210003 (2020).

11. V. S. Zarubin, G. N. Kuvyrkin, I. Y. Savelyeva, High Temperature, 53(2), pp. 234-239 (2015).

12. I. Yu. Savelyeva, Mechanics of Solids, 53(3), pp. 277-283 (2018).

13. G. N. Kuvyrkin, I. Y. Savelyeva, A. V. Zhuravsky, Mathematical Models and Computer Simulations, 10(3), pp. 299-307 (2018).

14. L. Segerlind, Application of the finite element method (1979).

15. M. P. Galanin, E. B. Savenkov, Numerical Analysis of Mathematical Models (2010). 